Research Paper

\title{
I7ß-Estradiol Inhibits Mesenchymal Stem Cells-Induced Human AGS Gastric Cancer Cell Mobility via Suppres- sion of CCL5- Src/Cas/Paxillin Signaling Pathway
}

\author{
Chao-Hung Kuo ${ }^{1,2,3}$, Chung-Jung Liu1,2, Chien-Yu Lu1,3, Huang-Ming Hu${ }^{1}$, Fu-Chen Kuo ${ }^{4}$, Yu-Sen Liou ${ }^{1}$, \\ Yuan-Chieh Yang ${ }^{5}$, Ming-Chia Hsieh 6 ,7, Oscar K. Lee ${ }^{8,9}$, Deng-Chyang Wu1,2,3,10, Sophie S.W. Wang1,2\#四, \\ Yao-Li Chen ${ }^{11,12 \# 冈 ~}$ \\ 1. Division of Gastroenterology, Department of Internal Medicine, Kaohsiung Medical University Hospital, Kaohsiung, Taiwan \\ 2. Cancer Center, Kaohsiung Medical University Hospital, Kaohsiung, Taiwan \\ 3. Department of Medicine, Faculty of Medicine, College of Medicine, Kaohsiung Medical University, Kaohsiung, Taiwan \\ 4. School of Medicine, College of Medicine, E-Da Hospital, I-Shou University, Kaohsiung, Taiwan \\ 5. Department of Laboratory Medicine, Kaohsiung Medical University Hospital, Kaohsiung, Taiwan \\ 6. Division of Endocrinology and Metabolism, Department of Internal Medicine, Changhua Christian Hospital, Changhua, Taiwan \\ 7. Graduate Institute of Integrated Medicine, China Medical University, Taichung, Taiwan \\ 8. Stem Cell Research Center, National Yang-Ming University, Taipei, Taiwan \\ 9. Department of Medical Research and Education, Taipei Veterans General Hospital, Taipei, Taiwan \\ 10. Division of Internal Medicine, Kaohsiung Municipal Hsiao-Kang Hospital, Kaohsiung Medical University, Kaohsiung, Taiwan \\ 11. Department of General Surgery, Changhua Christian Hospital, Changhua, Taiwan \\ 12. School of Medicine, Kaohsiung Medical University, Kaohsiung, Taiwan
}

\# These authors contributed equally to this paper.

$\triangle$ Corresponding authors: Yao-Li Chen, MD; Department of General Surgery, Changhua Christian Hospital, and School of Medicine, Kaohsiung Medical University, No. 135 Nan-Hsiao Street, Changhua City, Changhua County 500, Taiwan. Tel: +88647238595 ext4751 e-mail: 31560@cch.org.tw and Sophie S.W. Wang, MD, PhD; Division of Gastroenterology, Department of Internal Medicine, Kaohsiung Medical University Hospital, No. 100, Tz-You 1st Road, Kaohsiung City, 807 Taiwan Tel: + 88673121101ext7451; Fax: +88673135612 E-mail:swang910@gmail.com

(C) Ivyspring International Publisher. This is an open-access article distributed under the terms of the Creative Commons License (http://creativecommons.org/ licenses/by-nc-nd/3.0/). Reproduction is permitted for personal, noncommercial use, provided that the article is in whole, unmodified, and properly cited.

Received: 2013.06.07; Accepted: 2013.II.25; Published: 2013.12.10

\begin{abstract}
Gender differences in terms of mortality among many solid organ malignancies have been proved by epidemiological data. Estrogen has been suspected to cast a protective effect against cancer because of the lower mortality of gastric cancer in females and the benefits of hormone replacement therapy (HRT) in gastric cancer. Hence, it suggests that $17 \beta$-estradiol (E2) may affect the behavior of cancer cells. One of the key features of cancer-related mortality is metastasis. Accumulating evidences suggest that human bone marrow mesenchymal stem cells (HBMMSCs) and its secreted CCL-5 have a role in enhancing the metastatic potential of breast cancer cells. However, it is not clear whether E2 would affect HBMMSCs-induced mobility in gastric cancer cells. In this report, we show that CCL-5 secreted by HBMMSCs enhanced mobility in human AGS gastric cancer cells via activation of Src/Cas/Paxillin signaling pathway. Treatment with specific neutralizing antibody of CCL-5 significantly inhibited HBMMSCs-enhanced mobility in human AGS gastric cancer cells. We further observe that $17 \beta$-estradiol suppressed HBMMSCs-enhanced mobility by down-regulating CCL5-Src/Cas/paxillin signaling pathway in AGS cells. Collectively, these results suggest that $17 \beta$-estradiol treatment significantly inhibits HBMMSCS-induced mobility in human AGS gastric cancer cells.
\end{abstract}

Key words: Estrogen; Mesenchymal stem cell; Human gastric cancer cell; CCL-5; Cell mobility 


\section{Introduction}

Gastric cancer is one of the most common cancers $[1,2]$ and the secondary leading cause of cancer-related mortality worldwide [2]. Interestingly, gender differences in terms of incidence and mortality in gastric cancer have been observed, as in many other solid organ malignancies. Epidemiological data have shown that the incidence of gastric cancer in males is $\sim 2$-fold higher than in females worldwide [1, 3]. In addition, the delayed menopause $[4,5]$ and hormone replacement therapy (HRT) [3, 6] have been shown to reduce the risk and protect against gastric cancer in females. The same gender difference is also observed in the animal models. In the $N$-methyl- $N$ '-nitro-nitrosogunidine (MNNG)-induced gastric cancer, the incidence was reduced in female rats compared to the male counterparts $[7,8]$. Collectively, these findings suggest that the gender differences in gastric cancer may be associated with estrogen, which may offer the protective effect against the development and progression of gastric cancer.

Many studies have identified mesenchymal stem cells (MSCs) as an important cell subset which contributes to tumor progression and drug resistance [9, 10]. MSC has been shown to have the propensity of migrating to the damaged tissues. One of the key features of MSC is the capacity to secrete large amount of cytokines, chemokines, growth factors and to differentiate into tumor-associated fibroblasts [9, 11-13]. Consequently, MSC has been shown to promote tumor progression, including gastric tumor [14]. However, it is not clear whether estrogen has any protective influence on the effects of MSC on tumor progression.

Src family kinases (SFKs) have been involved in many biological activities during carcinogenesis, including cell adhesion, invasion, proliferation, and survival. Evidences suggest that the activities of SFK was up-regulated in human gastric carcinoma tissues [15]. As a result, targeting SFK may hold promise for the treatment of gastric cancer [16-18]. SFKs affect cell adhesion and migration through orchestrating the activation of its downstream Cas and paxillin scaffold proteins [19]. Src has been identified as a critical molecular factor in inducing cell adhesion and migration through activation of its downstream Cas and paxillin proteins, which contributes to the malignant development of human cancers [20-22]. Deregulation of Cas signaling is involved in gastric cancer progression and developmental defects in human[21]. Paxillin, a key regulator of the cellular migration machinery 2- and 3-dimensional culture, recently has been regarded as the future prognostic and therapeutic target[22, 23].
Hence, Src/Cas/Paxillin pathway is relevant in directing the migratory activity of gastric cancer cells.

In this study, we investigated whether $17 \beta$-estradiol (E2) treatment is sufficient to inhibit human bone marrow mesenchymal stem cells (HBMMSCs)-induced mobility in gastric cancer cells, and identified the underlying molecular mechanism in human gastric cancer cells.

\section{Methods}

\section{Antibodies, Reagents and Enzymes}

$17 \beta$-estradiol (E2) was purchased from Sigma (Sigma Chemical Co., St. Louis, Missouri, USA). The LY294002 (PI3K inhibitor), U0126 (MEK1/2 inhibitor), SB203680 (p38 MAPK inhibitor), SP600125 (JNK inhibitor), and PP2 (Src inhibitor) were purchased from TOCRIS (Ellisville, Missouri, USA). Human cytokine array kit (Proteome Profile ${ }^{\mathrm{TM}}$ Array) was purchased from R\&D system (Minneapolis, MN, USA). We utilized the following antibodies against Src, phospho-Src and phospho-Cas (Cell Signaling Technology, Inc. Beverly, MA, USA); Cas, phospho-paxillin and paxillin (Santa Cruz Biotechnology, Inc. Santa Cruz, California, USA).CCR5 and CCL-5 neutralizing antibody (R\&D; Minneapolis, MN, USA) was used for specifically blocking human CCL-5 function. Goat anti-mouse IgG antibody conjugated to horseradish peroxidase; goat anti-rabbit IgG antibody conjugated to horseradish peroxidase and rabbit anti-goat IgG horseradish peroxidase conjugate were purchased from Cell Signaling Technology, Inc. (Beverly, MA, USA). Normal goat IgG control was purchased from R\&D Systems (Minneapolis, MN, USA).

\section{Cell Culture}

Human bone marrow mesenchymal stem cells (HBMMSCs) were gifts from Dr. Oscar Kuang-Sheng Lee (National Yang-Ming University, Taipei, Taiwan). HBMMSCs were positive for CD29, CD44, CD73, CD90, and CD105, but were negative for CD13, CD34, CD45, and CD133. HBMMSCs were expanded and maintained in Iscove's modified Dulbecco's medium (IMDM, Gibco, Grand Island, NY, USA), supplemented with $10 \%$ fetal bovine serum (FBS, Hyclone, Logan, UT, USA), $10 \mathrm{ng} / \mathrm{ml}$ basic fibroblast growth factor (FGF-2; Sigma Chemical Co., St. Louis, Missouri, USA), $5 \mathrm{ng} / \mathrm{ml}$ epidermal growth factor (EGF; R\&D Systems, Minneapolis, MN, USA), 100 units penicillin, 1000 units streptomycin, and $2 \mathrm{mM}$ L-glutamine. HBMMSCs at passages 10-16 were used for the experiments in this study.

AGS, humnan gastric cancer cell line, purchased from the American Type Culture Collection (ATCC) (Rockville, MD, USA) were cultured on $100-\mathrm{mm}$ or 
60-mm culture dishes in RPMI-1640 medium supplemented with $100 \mu \mathrm{g} / \mathrm{ml}$ penicillin, $100 \mu \mathrm{g} / \mathrm{ml}$ streptomycin, $2 \mathrm{mM}$ glutamine, $1 \mathrm{mM}$ HEPS buffer, and $10 \%$ fetal bovine serum (all from Life Technologies, Carlsbad, CA, USA). Cell cultures were maintained at $37^{\circ} \mathrm{C}$ in a humidified $5 \% \mathrm{CO} 2$ atmosphere. Adherent cells were detached from the culture dishes with trypsin/EDTA (Life Technologies, Carlsbad, CA, USA).

\section{Human Cytokine Array}

Cell culture supernatant from HBMMSCs was collected by centrifuging at $10,000 \mathrm{~g}$ for $10 \mathrm{~min}$ to remove cell debris. The membranes from human protein cytokine array kit (Proteome Profiler ${ }^{\mathrm{TM}}$ Array; R\&D) were blocked with blocking buffer at room temperature for one hour, and were followed by incubation with cell culture supernates overnight at $2-8^{\circ} \mathrm{C}$ on a rocking platform. The membranes were then washed with wash buffer, and incubated with the diluted Streptavidin-HRP for 30 minutes at room temperature. After washing, the membranes were assayed by chemiluminescence.

\section{Immunoblotting}

To isolate total proteins, cultured AGS cells were washed with cold PBS and resuspended in lysis buffer (50 mM Tris , pH 7.5, 0.5M NaCl, 1.0 mM EDTA , pH $7.5,10 \%$ glycerol, $1 \mathrm{mM}$ BME, $1 \% \mathrm{NP} 40$ ) plus proteinase inhibitor cocktail and phosphatase inhibitor cocktail (Roche Molecular Biochemicals). After incubation for $30 \mathrm{~min}$ on ice, the supernatant was collected by centrifugation at $12,000 \mathrm{~g}$ for $15 \mathrm{~min}$ at $4{ }^{\circ} \mathrm{C}$, and the protein concentration was determined by the Bradford method. Sample containing equal proteins $(40 \mu \mathrm{g})$ were loaded and analyzed by Western blot analysis. Briefly, proteins were separated by $12 \%$ SDS-PAGE and transferred onto PVDF membrane (Life Technologies, Carlsbad, CA, USA). Membrane were blocked with blocking buffer (5\% non-fat dry milk, $20 \mathrm{mM}$ Tris- $\mathrm{HCl}, \mathrm{pH} 7.6,150 \mathrm{mM} \mathrm{NaCl}$, and $0.1 \%$ Tween 20 ) for at least $1 \mathrm{~h}$ at room temperature. Membranes were incubated with primary antibodies in the above solution on an orbit shaker at $4{ }^{\circ} \mathrm{C}$ overnight. Following primary antibody incubations, membranes were incubated with horseradish peroxidase-linked secondary antibodies (anti-rabbit, anti-mouse, or anti-goat IgG). Antibody-bound protein bands were detected using high sensitive Immobilon Western Chemiluminescent HRP Substrate (Millipore, Billerica, MA, USA), and photographed with Bio-Rad Chemiluminescence Imaging System (Bio-Rad Laboratories, Inc. Hercules, CA, USA)

\section{Cell Migration Assay}

Cell migration assay was performed by using the 24-well modified Boyden chambers containing membrane filter inserts with $8-\mu \mathrm{m}$ pores (Corning Incorporated Life Sciences, Tewksbury, MA, USA). Membrane filter inserts were pre-treated with collagen I (Sigma Chemical Co., St. Louis, Missouri, USA). To detect the effect of HBMMSCs on AGS cell mobility, HBMMSCs were plated in the lower chamber, whereas AGS cells were plated in the upper chamber. Serum- and phenol red- free medium and reagents in question, such as $17 \beta$-estradiol, recombinant CCL-5 protein or CCL-5 neutralizing antibody, were added to the assay system for $24 \mathrm{~h}$ or $48 \mathrm{~h}$ for experiments. At the end of experiments, cells on the bottom of membrane filter were fixed with $4 \%$ paraforrmaldehyde and stained with $1 \mu \mathrm{g} / \mathrm{ml}$ 4'-6-diamidine-2phenylindole dihydrochloride (DAPI; Roche Molecular Biochemicals) for $30 \mathrm{~min}$ to detect cell nucleus (blue stains) by fluorescence microscopy. The phenotype of migratory cells was evaluated by counting the cells under fluorescence microscopy at $40 \times$ and $100 \times$ magnifications, respectively.

\section{Total RNA Extraction}

Total RNA was isolated from cultured cells. The cells were homogenized in RNA lysis/binding buffer. The High Pure RNA Tissue Kit (Roche Applied Science, Mannheim, Germany) was used for RNA extraction.

\section{Reverse Transcription and Real-Time PCR Assay}

The standard reverse transcription and real-time PCR protocol was used in this study. For reverse transcription, the samples were incubated at $25^{\circ} \mathrm{C}$ for $10 \mathrm{~min}$, real-time PCR was initiated with a hot start (10 min at $95^{\circ} \mathrm{C}, 1$ cycle), the samples were then subjected to 40 cycles at $95{ }^{\circ} \mathrm{C}$ for $15 \mathrm{sec}$ and $60^{\circ} \mathrm{C}$ for 1 min. Data was analysed by StepOne real-time PCR system (Applied Biosystems, Foster City, California, USA). Primers were as follows: human CCL-5 forward primer CGCTG TCATC CTCAT TGCTA, reverse primer GCACT TGCCA CTGGT GTAGA; human beta-actin forward primer AAGAT ACCCA ATCAT TTTGA ACC, reverse primer AGCCA GTCCA ACGCA GAT (MISSION BIOTECH, Taipei, Taiwan).

\section{Enzyme-linked Immunosorbent Assay (ELISA)}

Conditioned medium (CM) from AGS cells culture, AGS cells/HBMMSCs co-culture, and HBMMSCs culture was collected by centrifuging at $10,000 \mathrm{~g}$ for $10 \mathrm{~min}$ to remove cell debris. The level of CCL-5 was measured using the ELISA kit obtained from 
R\&D Systems (Minneapolis, MN, USA) according to the manufacturer's instructions.

\section{Statistical Analysis}

Each experiment was duplicated for at least three times. Results were presented as the mean \pm standard deviation (SD). Statistical analysis was performed by using the Student's $t$ test. Significance was defined at the $\mathrm{p}<0.05\left({ }^{*}\right)$ or $\mathrm{p}<0.01\left({ }^{* *}\right)$ levels.

\section{Results}

\section{CCL-5 from human bone marrow mesen- chymal stem cells (HBMMSCs) enhanced mo- bility in human AGS cells}

To test whether HBMMSCs would induce mobility in AGS cells, the co-culture AGS/HBMMSC system in Boyden chamber assay was established. We found that HBMMSCs significantly enhanced mobility of human AGS gastric cancer cells. To identify which kind of soluble factor is responsible for AGS cell mobility, we further determine the soluble factors in the supernatant form HBMMSCs, using human cytokine protein array. The assay revealed that RANTES (CCL-5), interleukin-6 (IL-6), plasminogen activator inhibitor-1 (PAI-1; Serpin E1), interleukin-8 (IL-8), GRO $\alpha$ (CXCL-1), and macrophage migration inhibitory factor (MIF) were notably increased (data not shown). We then tested the role of CCL-5 in mediating the mobility of AGS cells, using the specific neutralizing antibody to eliminate the function of CCL-5 in the AGS/HBMMSC co-culture system. In-
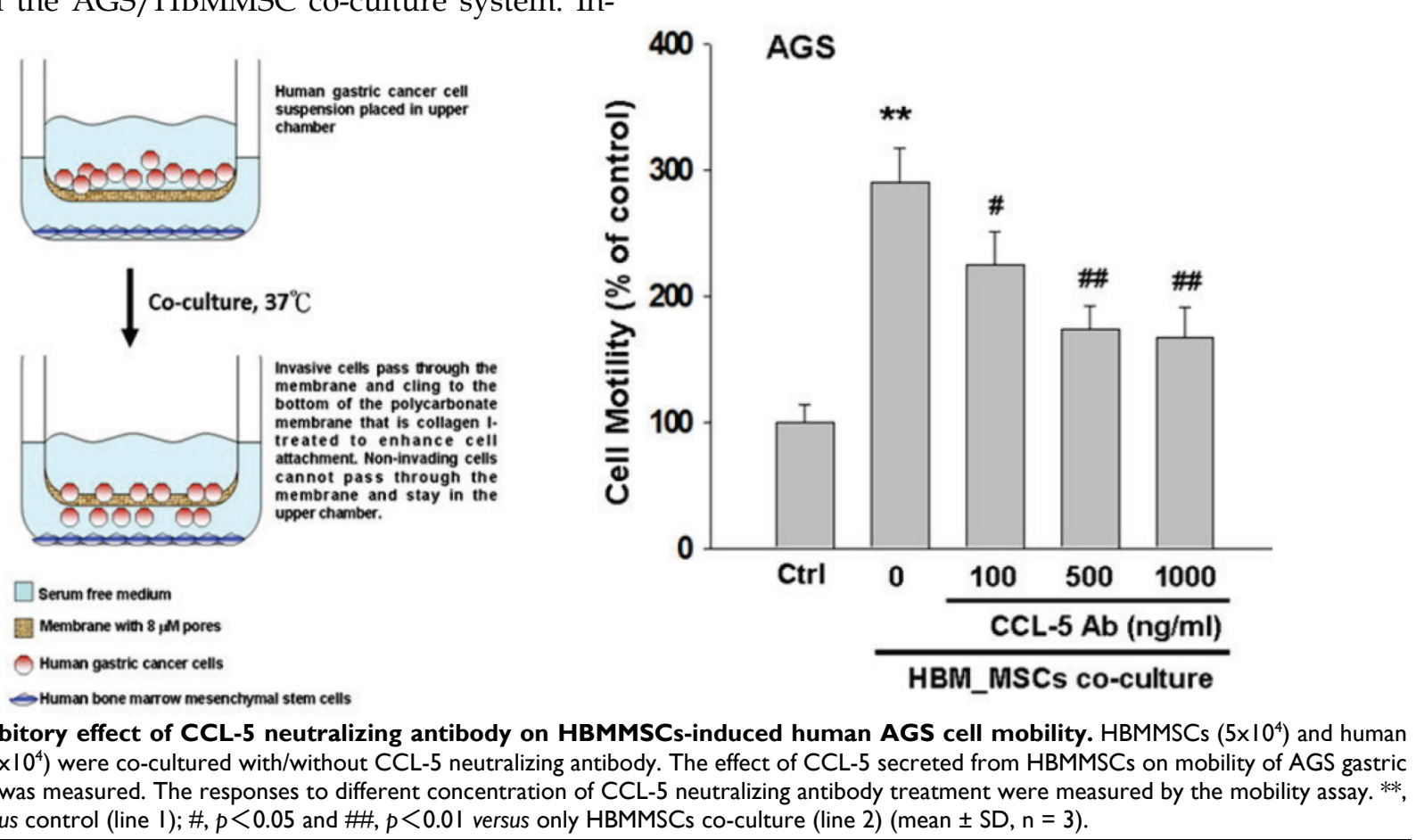

Fig. I. Inhibitory effect of CCL-5 neutralizing antibody on HBMMSCs-induced human AGS cell mobility. HBMMSCs $\left(5 \times 10^{4}\right)$ and human AGS cells $\left(5 \times 10^{4}\right)$ were co-cultured with/without CCL-5 neutralizing antibody. The effect of CCL-5 secreted from HBMMSCs on mobility of AGS gastric cancer cells was measured. The responses to different concentration of CCL- 5 neutralizing antibody treatment were measured by the mobility assay. $* *$, $p<0.01$ versus control (line I); $\#, p<0.05$ and \#, $p<0.01$ versus only HBMMSCs co-culture (line 2$)($ mean $\pm S D, n=3$ ). deed, the percentage of AGS cells migration was reduced by nearly $50 \%$ in the presence of CCL-5 neutralizing antibodies (Fig. 1). We further measured the concentration of CCL-5 in the supernatants that were form AGS cells alone, AGS cells/HBMMSCs co-culture, and HBMMSCs alone, respectively. The expression of CCL-5 was noted in HBMMSCs alone, and was increased in AGS cells/HBMMSCs co-culture (Fig. 2A). We further used quantitative reverse transcription-PCR to measure the expression of CCL-5 in AGS cells alone, AGS cells in co-culture, HBMMSCs in co-culture, and HBMMSCs alone. The findings showed that CCL-5 expression in HBMMSCs was remarkably higher than in AGS cells in this co-culture system (Fig. 2B). The data suggested that soluble CCL-5 protein may be mainly over-expressed from HBMMSCs in this co-culture (Fig. 2A). We also tested the effect of CCL-5 on AGS cells that were treated with HBMMSCs supernatant in the presence of CCL-5 specific neutralizing antibody. The capacity of AGS cell migration was significantly reduced by CCL-5 specific neutralizing antibody (Fig. 2C).

To further confirm the role of CCL-5 in mediating mobility in AGS cells, we treated AGS cells with recombinant CCL-5 $(0,1,10,20,50$ and $100 \mathrm{ng} / \mathrm{ml})$ for 24 hours. We observed that low level of CCL-5 (1 $\mathrm{ng} / \mathrm{ml}$ ) was enough to enhance the migration of AGS cells by $100 \%$. (Fig. 3) Collectively, CCL-5 secreted from HBMMSCs might mediate the mobility in AGS gastric cancer cells. 
A

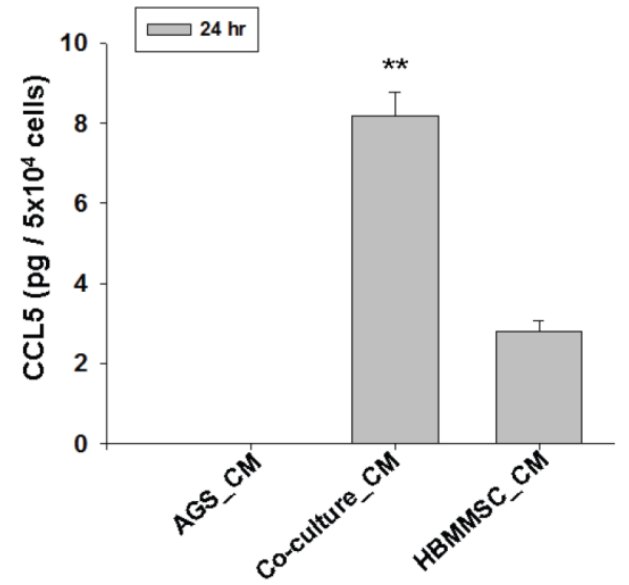

B

Real-time qRT-PCR

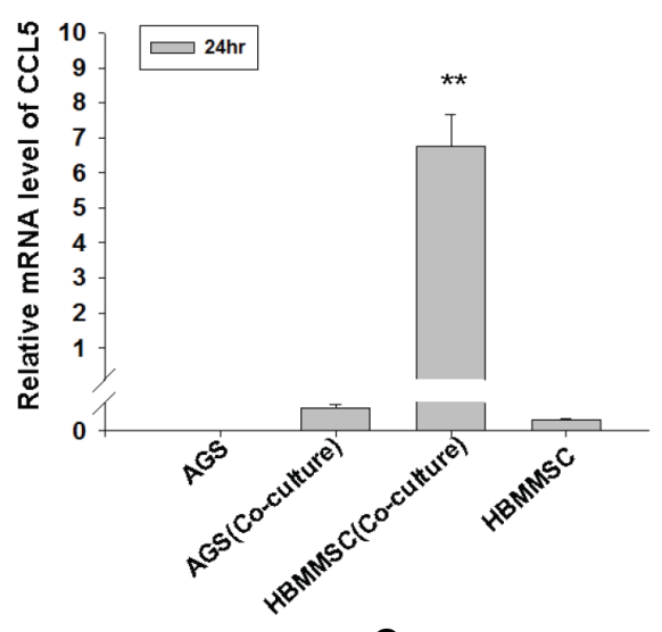

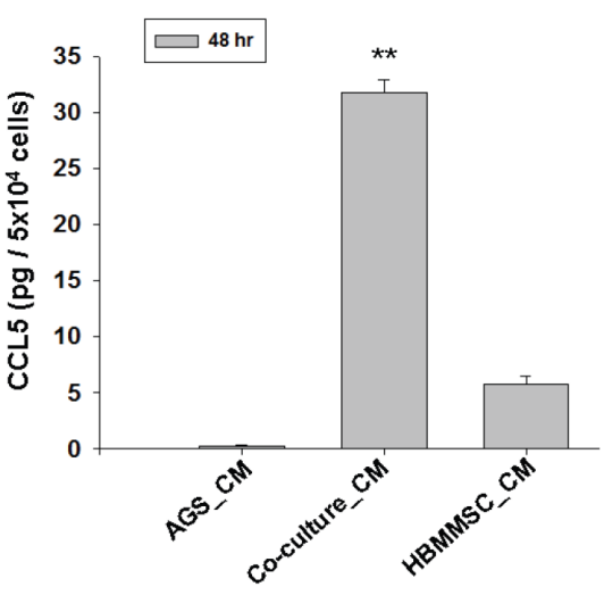

Real-time qRT-PCR

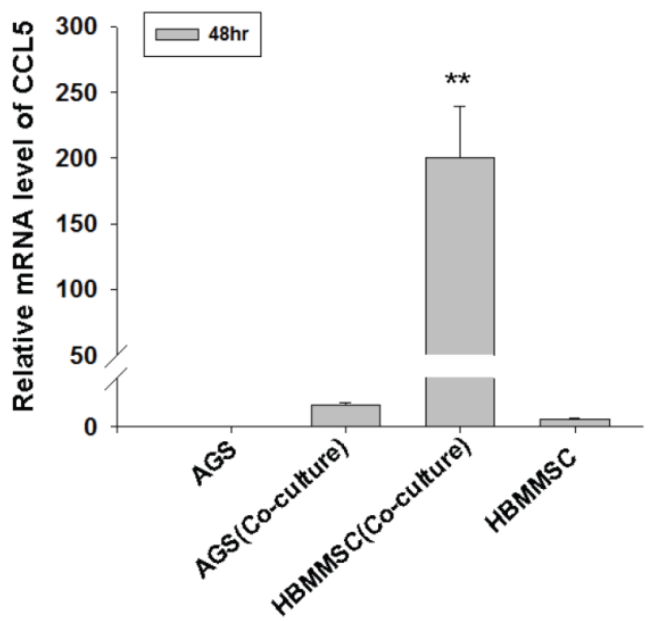

C

AGS

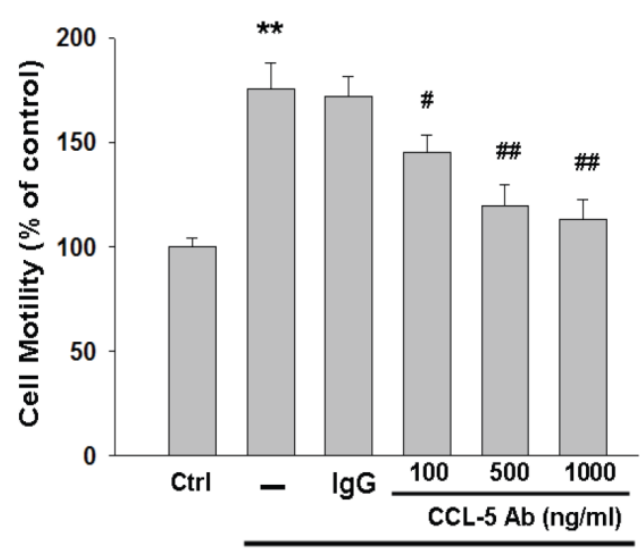

HBM_MSCs supernatant

Fig. 2. Increased CCL-5 expression by HBMMSCs in AGS/HBMMSC co-culture system. (A) Enzyme-linked immunosorbent assay for CCL-5 concentration in AGS cells $\left(5 \times 10^{4}\right)$ alone, AGS cells $\left(5 \times 10^{4}\right) / \mathrm{HBMMSCs}\left(5 \times 10^{4}\right)$, and $\mathrm{HBMMSCs}\left(5 \times 10^{4}\right)$. (B) Quantitative reverse transcription PCR for relative CCL-5 mRNA level to beta-actin in AGS cells alone, AGS cells in co-culture, HBMMSCs in co-culture, and HBMMSCs alone. (C) The effect of CCL-5 from supernatant of HBMMSCs on mobility of AGS gastric cancer cells was measured. **, $p<0.01$ versus control; \#, $p<0.05$ and \#, $p<0.01$ versus only HBMMSCs co-culture (line 2) (mean $\pm \mathrm{SD}, \mathrm{n}=3$ ). 


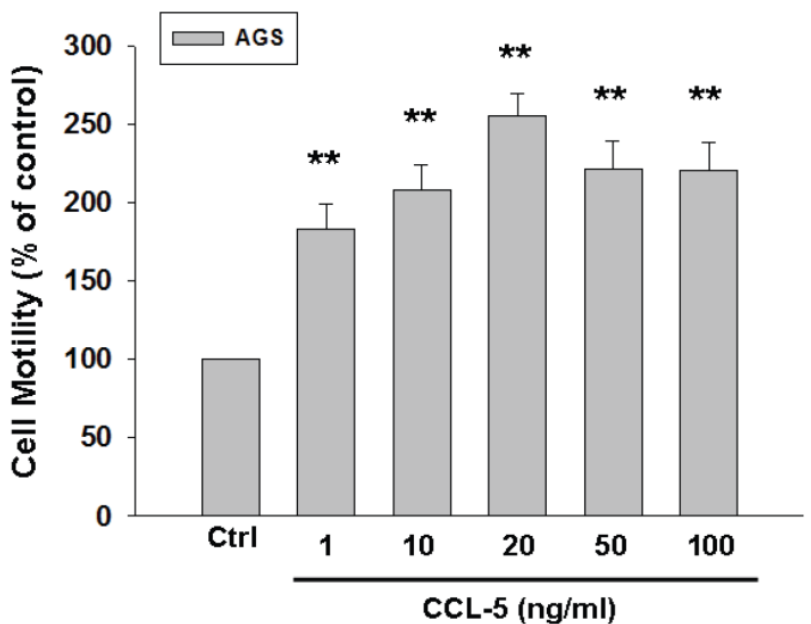

Fig. 3. Recombinant CCL-5 increases human AGS cell mobility. Human AGS cells were treated with recombinant CCL-5 (I, I0, 20, 50 and $100 \mathrm{ng} / \mathrm{ml}$ ) for $24 \mathrm{~h}$, and subsequently measured the capacity of cell mobility. The responses to different concentration of CCL-5 treatment were measured by mobility assay. ${ }^{* *}, p<0.0$ I versus control (line I) (mean $\pm S D$, $n=3)$.

\section{Src mediated CCL-5-induced mobility in AGS cells}

To identify the signaling pathway(s) involved in the CCL-5-regulated mobility in AGS cells, we applied the following inhibitors, such as LY294002 (Akt activation inhibitor), U0126 (ERK1/2 activation inhibitor), SB203580 (p38 MAPK inhibitor), SP600125 (JNK1/2 inhibitor), and PP2 (Src activation inhibitor), to block the corresponding pathways in the presence of CCL-5. AGS cells were pre-incubated with LY294002 (1 $\mu \mathrm{M})$, U0126 (1 $\mu \mathrm{M})$, SB203580 (1 $\mu \mathrm{M})$, SP600125 $(1 \mu \mathrm{M})$ or PP2 $(1 \mu \mathrm{M})$ for 1hour, followed by the administration of CCL-5 (10ng/ml) for 24 hours. After treatments, AGS cells were subjected to the mobility assay. We found that Src inhibitor (PP2) completely abolished CCL-5-induced mobility in AGS cells (Fig. 4A). We also measured the effect of these inhibitors in the absence of CCL- 5 soluble protein. The finding suggested that Src indeed play a critical role in regulating the mobility in AGS cells (Fig. 4B). It suggested that CCL-5 might mediate the mobility in human AGS gastric cancer cells via Src signaling pathway.

\section{7 $\beta$-estradiol inhibited CCL-5-induced AGS cell mobility by suppressing CCL-5/Src/Cas/Paxillin activation}

To test the effect of $17 \beta$-estradiol (E2) on HBMMSCs-induced mobility in human AGS gastric cancer cells, $17 \beta$-estradiol (E2) was added to the co-culture system for $24 \mathrm{~h}$ and $48 \mathrm{~h}$. The finding showed that, after 24 hours, E2 $\left(10^{-8} \mathrm{M}\right)$ completely suppressed HBMMSCs-induced mobility in AGS cells
(AGS $=100 \pm 13.747$;

AGS+MSC $=233.758 \pm 41.479$; AGS+MSC+E2 $=102 \pm 10.238$ ). Although the suppression effect of $\mathrm{E} 2$ was attenuated after 48 hours (AGS = 167.516 $\pm 21.465 ;$ AGS + MSC $=705.733 \pm 92.366 ;$ AGS + MSC $+\mathrm{E} 2=325.438 \pm 36.478)$, the percentage of HBMMSCs-induced migratory cells was suppressed by $50 \%$ (Fig. $5 \mathrm{~A})$. The effect of E2 $\left(10^{-8} \mathrm{M}\right)$ alone on AGS cell migration was also measured. We found that E2 $\left(10^{-8} \mathrm{M}\right)$ treatment significantly reduced AGS cell migration without influencing CCR5 protein expression (Fig. 5B, C).

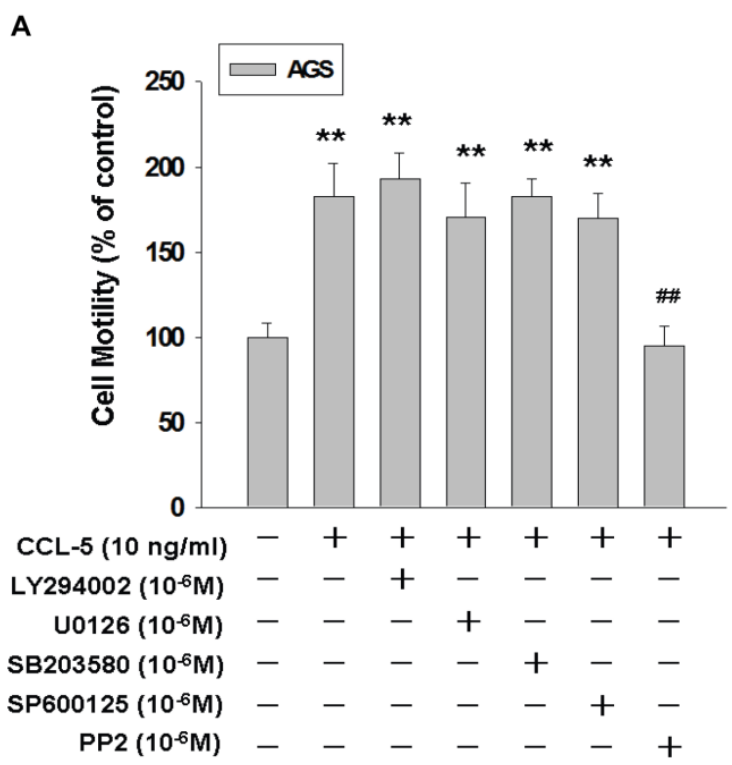

B

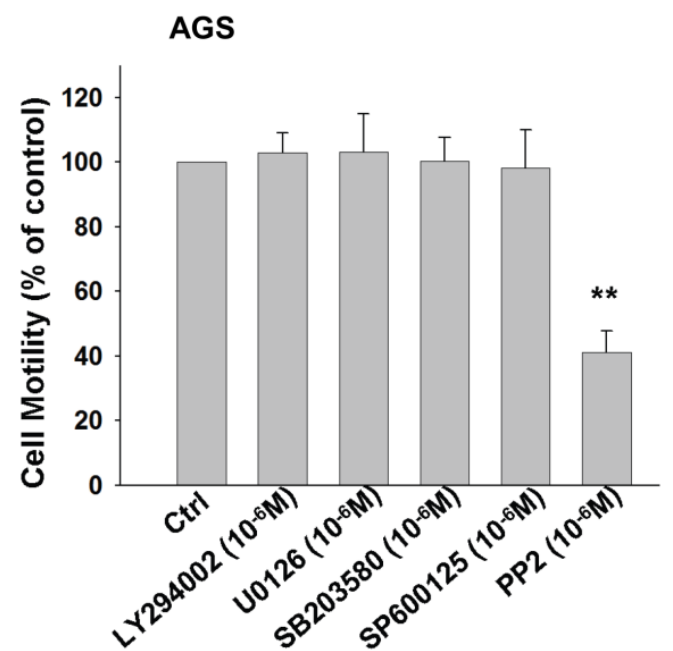

Fig. 4. CCL-5 induces AGS cell mobility via Src signaling pathway. (A) AGS cells were pretreated with vehicle, LY294002 (Akt activation inhibitor, I $\mu \mathrm{M}$ ), U0I 26 (ERKI/2 activation inhibitor, I $\mu \mathrm{M}$ ), SB203580 (p38 MAPK inhibitor, I $\mu$ M), SP600I 25 (JNKI/2 inhibitor, I $\mu$ M) or PP2 (Src inhibitor, $I \mu \mathrm{M})$ for $\mathrm{Ih}$, and followed by recombinant CCL-5 $(10 \mathrm{ng} / \mathrm{ml})$ administration for 24h. (B) AGS cells were treated with these inhibitors, respectively. AGS cells were harvested and measured for cellular mobility capacity. **, $p<0.01$ versus control (line I); \#, $p<0.01$ versus CCL-5 treatment (line 2) (mean $\pm S D, n=3)$. 
To further investigate the molecular mechanism underlying the E2 inhibitory effect on CCL-5-induced AGS cell migration, we treated AGS cells with CCL-5 $(1 \mathrm{ng} / \mathrm{ml})$ for 3 hours and measured the phosphorylation/activation of cell mobility-related proteins by immunoblotting assay. Phosphorylation of Src, Cas and paxillin were significantly induced in response to CCL-5 stimulation. The findings suggested that activation of Src/Cas/paxillin by CCL-5 might play an important role in mediating HBMMSCs-induced mobility in AGS cells. More importantly, $17 \beta$-estradiol (E2; $10^{-8}$ and $\left.10^{-9} \mathrm{M}\right)$ significantly inhibited A

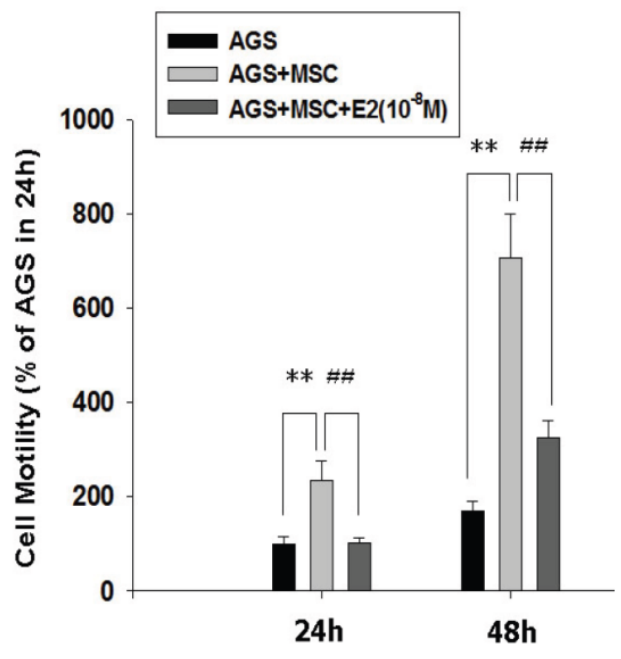

CCL-5-induced phosphorylation of Src, Cas and paxillin in human AGS gastric cancer cells. The activation status of Src, Cas and paxillin depends on the ratio of the phosphorylated and total proteins. As a result, we observed a nearly elimination of phosphor-Src (Tyr416), phospho-Cas (Tyr165, and phospho-paxillin (Tyr118) after E2 administration. Therefore, these data confirmed that $17 \beta$-estradiol significantly inhibited CCL-5/Src/Cas/Paxillin-induced mobility in AGS cells (Fig. 6).

B

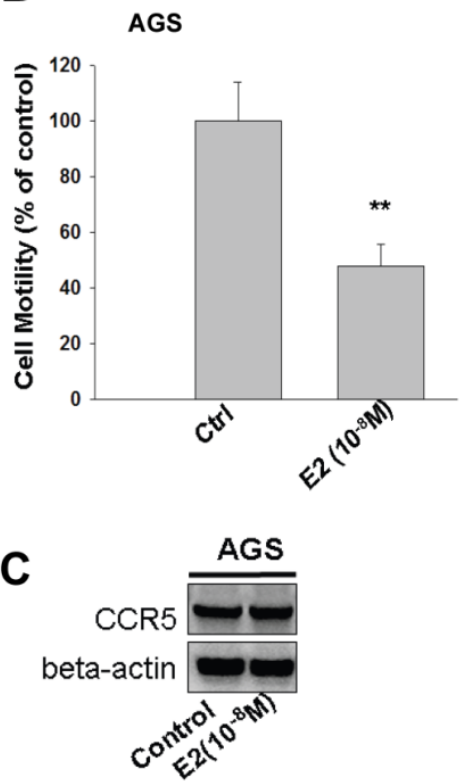

Fig. 5. Inhibition of HBMMSCs-induced cellular mobility by $17 \beta$-estradiol in human AGS gastric cancer cells. $\mathrm{HBMMSCs}\left(5 \times 10^{4}\right)$ and human AGS cells $\left(5 \times 10^{4}\right)$ were co-culture with/without $17 \beta$-estradiol $\left(10^{-8} \mathrm{M}\right)$ treatment for $24 \mathrm{~h}$ and $48 \mathrm{~h}$. The effect of I $7 \beta$-estradiol on HBMMSCs-induced cellular mobility in human AGS gastric cancer cells was measured. The effect of $17 \beta$-estradiol $\left(10^{-8} \mathrm{M}\right)$ alone on mobility and CCR5 protein expression in AGS cells was measured by cell migration assay and immunoblotting. ${ }^{* *}, p<0.0$ I versus control; \#\#, $p<0.0$ I versus only HBMMSCs co-culture (mean \pm SD, $\mathrm{n}=3$ ).
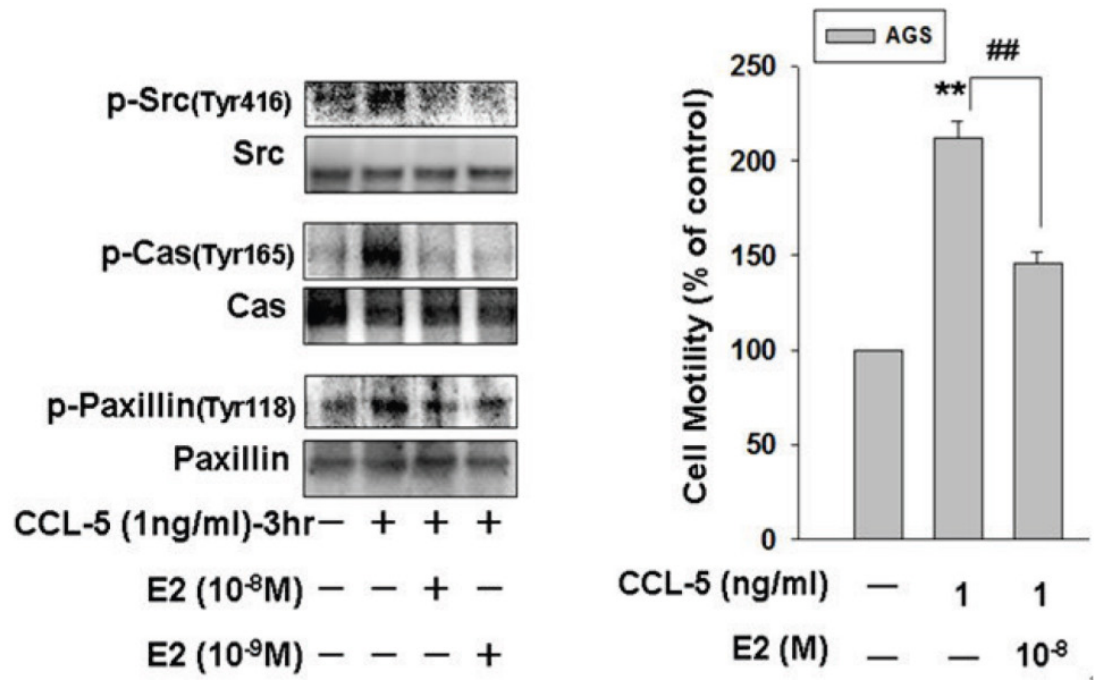

Fig. 6. I7 $\beta$-estradiol down-regulates CCL-5-induced mobility activity by suppressing Src/Cas/Paxillin activation in human AGS cells. Human AGS cells were pre-treated with $17 \beta$-estradiol $\left(10^{-8}\right.$ and $\left.10^{-9} \mathrm{M}\right)$ for $\mathrm{lh}$, and followed by CCL-5 $(\mathrm{I} \mathrm{ng} / \mathrm{ml})$ treatment for $24 \mathrm{~h}$. AGS cells were then harvested for immunoblotting assay (A), and measuring the capacity of cell mobility (B). ${ }^{* *}, p<0.0 \mathrm{I}$ versus control (line I); $\#, p<0.0 \mathrm{I}$ versus CCL-5 treatment (line 2) (mean $\pm S D, n=3)$. 


\section{ESTROGEN}

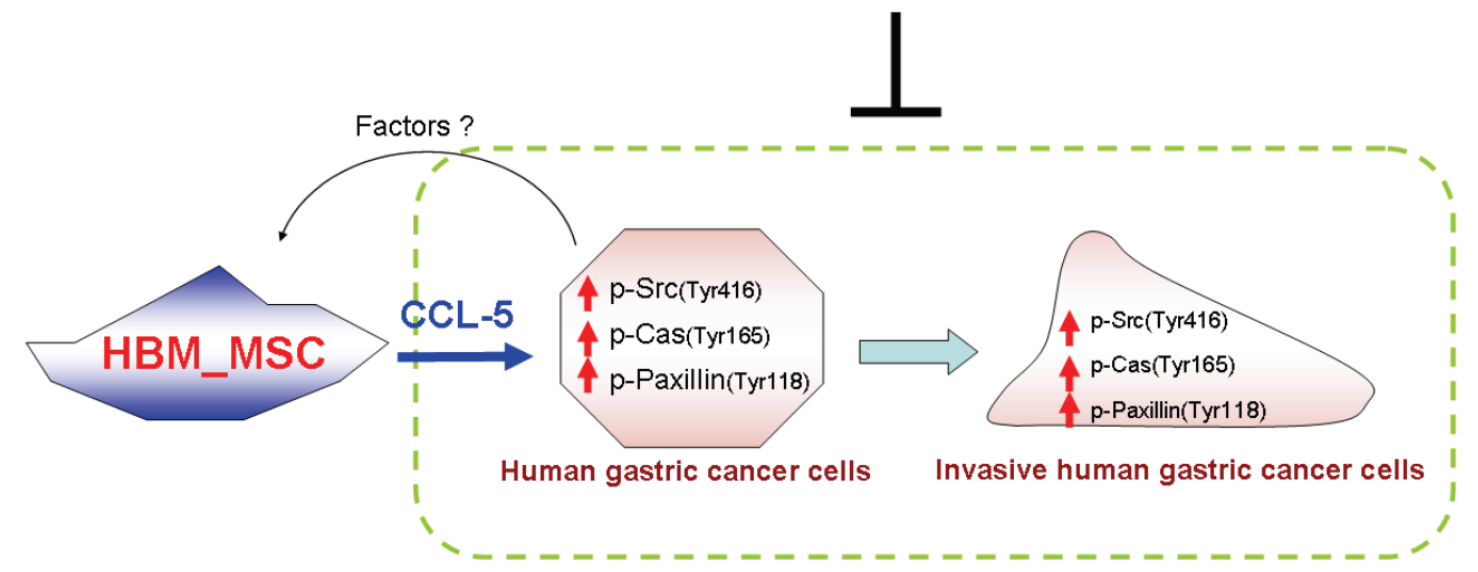

Fig. 7. A schematic representation showing $17 \beta$-estradiol inhibition of cell mobility via suppression of CCL5 - Src/Cas/Paxillin pathways in human AGS gastric cancer cells. CCL-5 is mainly secreted from HBMMSCs, which is great enhanced when co-culturing with AGS cells. Soluble CCL-5 induces the activation of Src, Cas and paxillin, then contributing to the changes in organization of cellular actin cytoskeleton, which thus promotes cell mobility events in human AGS gastric cancer cells. Treatment of $17 \beta$-estradiol significantly inhibits HBMMSCs-induced cell mobility by suppressing activation of CCL5-Src/Cas/paxillin signaling pathway.

\section{Discussion}

Human bone-marrow-derived mesenchymal stem cells (HBMMSCs) have been shown to enhance the metastatic potential of breast cancer cells via de novo secretion of the CCL-5 and through the chemokine receptor CCR5 [24]. Some studies showed that CCL-5 and CCL-5 receptors (CCR1, CCR3 and CCR5) are expression in gastric tissue and cell lines. Tumor over-expression of CCL-5 and CCR5 are associated with a lower survival rate in patients with gastric cancer $[25,26]$. However, whether CCL-5/CCR5 signaling is capable of enhancing cell mobility in other solid organ cancer cells and which the downstream signaling pathways of CCL-5/CCR5 are involved remain unclear. In this study, we confirmed that CCL-5 secreted from HBMMSCs enhanced mobility in human gastric cancer AGS cells. CCL-5 specific neutralizing antibody was able to suppress CCL-5-enhanced AGS cell mobility by blocking CCL-5 function. In addition, we also confirmed that $\mathrm{Src} / \mathrm{Cas} /$ Paxillin signaling pathway mainly mediated CCL-5-enhanced mobility in AGS cells. More importantly, we showed that $17 \beta$-estradiol (E2) $\left(10^{-8}\right.$ and $\left.10^{-9} \mathrm{M}\right)$ suppressed CCL-5-mediated mobility activity in AGS cells by down-regulating Src/Cas/Paxillin pathway (Fig. 7).

Mesenchymal stem cells (MSCs) recently have attracted attentions due to their capacity of migrating to and engrafting into tumor microenvironment [14, 27]. As a result, MSCs have offered a great opportunity to understand the intricate tumor microenvironment. Studies have showed that MSCs can promote tumor growth by migrating to the developing intrahepatic cholangiocarcinoma via SFD-1 $\alpha$ /CXCR4 signaling pathway[28], and to human medulloblastoma via MMP2 [29]. MSCs have been suggested as a source of cancer associated fibroblasts (CAF), which, in turn, promotes tumor growth and metastasis. Tumor-derived osteopontin (OPN) was shown to promote CCL-5 secretion from MSCs and greatly increased metastasis capacity in breast cancer[30]. Consequently, the role of MSC and CCL-5 in terms of promoting tumor metastasis has been emphasized. However, whether it is applicable in the context of gastric cancer is not clear. In this study, we observed CCL-5-secreted by HBMMSCs enhanced mobility in human gastric cancer AGS cells, and CCL-5 specific neutralizing antibody eliminated this enhanced mobility. Hence, we can confirme that HBMMSC-secreted CCL-5 can also enhance migration of human AGS gastric cancer cells.

The molecular mechanisms underlying gastric carcinogenesis is complicated [31]. Most of the well-known signaling pathways are also involved, such as phosphatidylinositide 3-kinase (PI3-K)/Akt (protein kinase B) and mitogen-activated protein kinases (MAPKs) pathways and its three major subfamilies, such as the extracellularly responsive kinases (ERKs), the c-Jun N-terminal kinases (JNKs), also known as stress-activated protein kinases (SAPKs), and the p38 MAPKs [31,32]. Another pathway that has received attentions is Src pathway. Given its critical role in the variety of cellular regulations, such as cell division, mobility, adhesion, angiogenesis, and survival, Src is regarded as the attractive target for future anti-cancer therapeutics [33]. Src kinase closely regulates the phosphorylation and activation of Cas 
adhesion protein and paxillin cytoskeletal protein, which regulate cell migration in invasive cells during cancer metastasis [16-18]. In this study, we observed that phosphorylation/activation of Src, Cas and paxillin were induced by CCL-5 stimulation (Fig. 6). The critical role of Src was confirmed by using PP2, a specific inhibitor for Src, in the mobility assay. Compared with various signaling pathway inhibitors including LY294002 (PI3K/Akt activation inhibitor), U0126 (ERK1/2 activation inhibitor), SB203580 (p38 MAPK inhibitor), and SP600125 (JNK1/2 inhibitor), the findings indicated that Src mainly mediatesd CCL-5-upregulated mobility activity in human AGS gastric cancer cells and highlighted the role of Src in cell migration and cancer metastasis.

Another important finding in this report is the protective effect of estrogen in cell migreation. Protective properties of estrogen against gastric cancer development have been reported because of the reduced gastric cancer risk in the delayed menopausal women $[4,5]$, and in the hormone replacement therapy (HRT)-treated women compared with non-treated counterparts [3, 6, 34]. In Helicobacter pylori (H. pylori)-infected INS-GAS male mice model, 17ß-estradiol (E2) prevented H. pylori-induced gastric cancer by reducing leukocyte recruitment and down-regulating oncogenic signaling pathways [35]. In the present study, we observed that $17 \beta$-estradiol treatment significantly suppressed HBMMSCS-induced mobility in human AGS gastric cancer cells. The 17 $\beta$-estradiol suppression of HBMMSCS-induced cellular mobility may be through the down-regulation of CCL5-Src/Cas/paxillin signaling pathway.

\section{Abbreviations}

E2, 17ß-estradiol; HBMMSCs, Human bone marrow mesenchymal stem cells; SFKs, Src family kinases; IL-6, interleukin-6; IL-8, interleukin-8; MIF, macrophage migration inhibitory factor; PAI-1, plasminogen activator inhibitor-1; PKB/Akt, Protein kinase B; PI3-K, phosphatidylinositol 3-kinase; ERK, extracellular signal regulated kinase; p38 MAPK, p38 mitogen-activated protein kinase; JNK, c-Jun N-terminal kinase; ECM, extracellular matrix; PBS, phosphate-buffered saline.

\section{Acknowledgement}

The authors thank the supports from Excellence for cancer research center grant, DOH102-TD-C-111-002, Department of Health, Executive Yuan, Taiwan, ROC, Kaohsiung Medical University Hospital (KMUH100-0I01, , KMUH98-8G63), National Science Council (NSC101-2811-B-037-008, NSC-2314-B-037-052-MY3) and Changhua Christian
Hospital-Kaohsiung Medical University (100-CCHKMU-005)

\section{Competing Interests}

The authors have declared that no competing interest exists.

\section{References}

1. Parkin DM, Bray F, Ferlay J, Pisani P. Global cancer statistics, 2002. CA: a cancer journal for clinicians. 2005; 55: 74-108.

2. Crew KD, Neugut AI. Epidemiology of gastric cancer. World J Gastroenterol. 2006; 12: 354-62.

3. Chandanos E, Lagergren J. Oestrogen and the enigmatic male predominance of gastric cancer. Eur J Cancer. 2008; 44: 2397-403.

4. Frise S, Kreiger N, Gallinger S, Tomlinson G, Cotterchio M. Menstrual and reproductive risk factors and risk for gastric adenocarcinoma in women: findings from the canadian national enhanced cancer surveillance system. Annals of epidemiology. 2006; 16: 908-16.

5. Freedman ND, Chow WH, Gao YT, Shu XO, Ji BT, Yang G, et al. Menstrual and reproductive factors and gastric cancer risk in a large prospective study of women. Gut. 2007; 56: 1671-7.

6. Camargo MC, Goto Y, Zabaleta J, Morgan DR, Correa P, Rabkin CS. Sex hormones, hormonal interventions, and gastric cancer risk: a meta-analysis. Cancer Epidemiol Biomarkers Prev. 2012; 21: 20-38.

7. Furukawa H, Iwanaga T, Koyama H, Taniguchi H. Effect of sex hormones on the experimental induction of cancer in rat stomach - a preliminary study. Digestion. 1982; 23: 151-5.

8. Furukawa H, Iwanaga T, Koyama H, Taniguchi $\mathrm{H}$. Effect of sex hormones on carcinogenesis in the stomachs of rats. Cancer Res. 1982; 42: 5181-2.

9. Loebinger MR, Kyrtatos PG, Turmaine M, Price AN, Pankhurst Q, Lythgoe MF, et al. Magnetic resonance imaging of mesenchymal stem cells homing to pulmonary metastases using biocompatible magnetic nanoparticles. Cancer research. 2009; 69: 8862-7.

10. Meads MB, Hazlehurst LA, Dalton WS. The bone marrow microenvironment as a tumor sanctuary and contributor to drug resistance. Clin Cancer Res. 2008; $14:$ 2519-26.

11. Dawson MR, Chae SS, Jain RK, Duda DG. Direct evidence for lineage-dependent effects of bone marrow stromal cells on tumor progression. American journal of cancer research. 2011; 1: 144-54.

12. Hung SC, Pochampally RR, Chen SC, Hsu SC, Prockop DJ. Angiogenic effects of human multipotent stromal cell conditioned medium activate the PI3K-Akt pathway in hypoxic endothelial cells to inhibit apoptosis, increase survival, and stimulate angiogenesis. Stem cells (Dayton, Ohio). 2007; 25: 2363-70.

13. Mishra PJ, Mishra PJ, Humeniuk R, Medina DJ, Alexe G, Mesirov JP, et al. Carcinoma-associated fibroblast-like differentiation of human mesenchymal stem cells. Cancer research. 2008; 68: 4331-9.

14. Houghton J, Stoicov C, Nomura S, Rogers AB, Carlson J, Li H, et al. Gastric cancer originating from bone marrow-derived cells. Science. 2004; 306: 1568-71.

15. Takekura N, Yasui W, Yoshida K, Tsujino T, Nakayama H, Kameda T, et al. pp60c-src protein kinase activity in human gastric carcinomas. International journal of cancer. 1990; 45: 847-51.

16. Okamoto W, Okamoto I, Yoshida T, Okamoto K, Takezawa K, Hatashita E, et al. Identification of c-Src as a potential therapeutic target for gastric cancer and of MET activation as a cause of resistance to c-Src inhibition. Molecular cancer therapeutics. 2010; 9: 1188-97.

17. Nam HJ, Im SA, Oh DY, Elvin P, Kim HP, Yoon YK, et al. Antitumor activity of saracatinib (AZD0530), a c-Src/Abl kinase inhibitor, alone or in combination with chemotherapeutic agents in gastric cancer. Molecular cancer therapeutics. 2013; 12: 16-26.

18. Kim LC, Song L, Haura EB. Src kinases as therapeutic targets for cancer. Nature reviews. 2009; 6: 587-95.

19. Tsuda M, Tanaka S. Roles for crk in cancer metastasis and invasion. Genes \& cancer. 2012; 3: 334-40.

20. Tsuda M, Tanaka S. Roles for crk in cancer metastasis and invasion. Genes Cancer. 2012; 3: 334-40.

21. Noguchi T, Tsuda M, Takeda H, Takada T, Inagaki K, Yamao T, et al. Inhibition of cell growth and spreading by stomach cancer-associated protein-tyrosine phosphatase-1 (SAP-1) through dephosphorylation of p130cas. The Journal of biological chemistry. 2001; 276: 15216-24.

22. Deakin NO, Pignatelli J, Turner CE. Diverse roles for the paxillin family of proteins in cancer. Genes Cancer. 2012; 3: 362-70.

23. Deakin NO, Pignatelli J, Turner CE. Diverse roles for the paxillin family of proteins in cancer. Genes \& cancer. 2012; 3: 362-70.

24. Karnoub AE, Dash AB, Vo AP, Sullivan A, Brooks MW, Bell GW, et al. Mesenchymal stem cells within tumour stroma promote breast cancer metastasis. Nature. 2007; 449: 557-63

25. Sugasawa H, Ichikura T, Tsujimoto H, Kinoshita M, Morita D, Ono S, et al. Prognostic significance of expression of CCL5/RANTES receptors in patients with gastric cancer. Journal of surgical oncology. 2008; 97: 445-50. 
26. Kim HK, Song KS, Park YS, Kang YH, Lee YJ, Lee KR, et al. Elevated levels of circulating platelet microparticles, VEGF, IL-6 and RANTES in patients with gastric cancer: possible role of a metastasis predictor. Eur J Cancer; 2003;: 184-91.

27. Takaishi S, Okumura T, Wang TC. Gastric cancer stem cells. J Clin Oncol. 2008; 26: $2876-82$.

28. Okamoto K, Tajima H, Nakanuma S, Sakai S, Makino I, Kinoshita J, et al. Angiotensin II enhances epithelial-to-mesenchymal transition through the interaction between activated hepatic stellate cells and the stromal cell-derived factor-1/CXCR4 axis in intrahepatic cholangiocarcinoma. International journal of oncology. 2012; 41: 573-82.

29. Bhoopathi P, Chetty C, Gogineni VR, Gujrati M, Dinh DH, Rao JS, et al. MMP-2 mediates mesenchymal stem cell tropism towards medulloblastoma tumors. Gene therapy. 2011; 18: 692-701.

30. Mi Z, Bhattacharya SD, Kim VM, Guo H, Talbot LJ, Kuo PC. Osteopontin promotes CCL5-mesenchymal stromal cell-mediated breast cancer metastasis. Carcinogenesis. 2011; 32: 477-87.

31. Adachi $Y$, Yamamoto $H$, Ohashi $H$, Endo $T$, Carbone DP, Imai $K$, et al. A candidate targeting molecule of insulin-like growth factor-I receptor for gastrointestinal cancers. World J Gastroenterol. 2010; 16: 5779-89.

32. Cargnello M, Roux PP. Activation and function of the MAPKs and their substrates, the MAPK-activated protein kinases. Microbiol Mol Biol Rev. 2011; 75: 50-83.

33. Summy JM, Gallick GE. Src family kinases in tumor progression and metastasis. Cancer metastasis reviews. 2003; 22: 337-58.

34. Lindblad M, Garcia Rodriguez LA, Chandanos E, Lagergren J. Hormone replacement therapy and risks of oesophageal and gastric adenocarcinomas. British journal of cancer. 2006; 94: 136-41.

35. Sheh A, Ge Z, Parry NM, Muthupalani S, Rager JE, Raczynski AR, et al. 17beta-estradiol and tamoxifen prevent gastric cancer by modulating leukocyte recruitment and oncogenic pathways in Helicobacter pylori-infected INS-GAS male mice. Cancer prevention research (Phila). 2011; 4: 1426-35. 